\title{
Neue orale Therapieoption bei Psoriasis
}

\author{
Hoffnungen setzt man bei der Psoriasis-Behandlung auf Januskinase-Inhibitoren. Sie werden im \\ Gegensatz zu anderen Biologika oral eingenommen. In einer Phase-3-Studie zeigte ein solcher \\ Inhibitor eine vergleichbare Wirksamkeit und Sicherheit wie ein Biologikum.
}

\begin{abstract}
Der orale Januskinase(JAK)-Inhibitor Tofacitinib bewies in einer randomisierten, doppelblinden Phase-3-Studie in der 10-mg-Dosis seine Nichtunterlegenheit gegenüber dem Biologikum Etanercept und seine Überlegenheit gegenüber Placebo bei Patienten mit chronischer mittelschwerer bis schwerer Plaque-Psoriasis. Damit könne diese Substanz künftig eine geeignete und gut verträgliche Therapieoption für diese Patienten werden, berichten die Studienautoren um Hervé Bachelez vom Saint-Louis Hospital in Paris in „The Lancet". Tofacitinib greift durch den JAK/STAT-Signalweg an der Signaltransduktion vieler Zytokine an. In den USA ist das Medikament bisher zur Therapie erwachsener Patienten mit moderater bis schwerer aktiver Rheumatoider Arthritis (RA) seit 2012 zugelassen. In Europa wurde die Zulassung von der europäischen Arzneimittelbehörde 2013 abgelehnt.
\end{abstract}

\section{0-mg-Dosis gegenüber Placebo überlegen}

1.106 Patienten aus 122 dermatologischen Zentren weltweit konnten die Wissenschaftler für die von der Firma Pfizer finan-

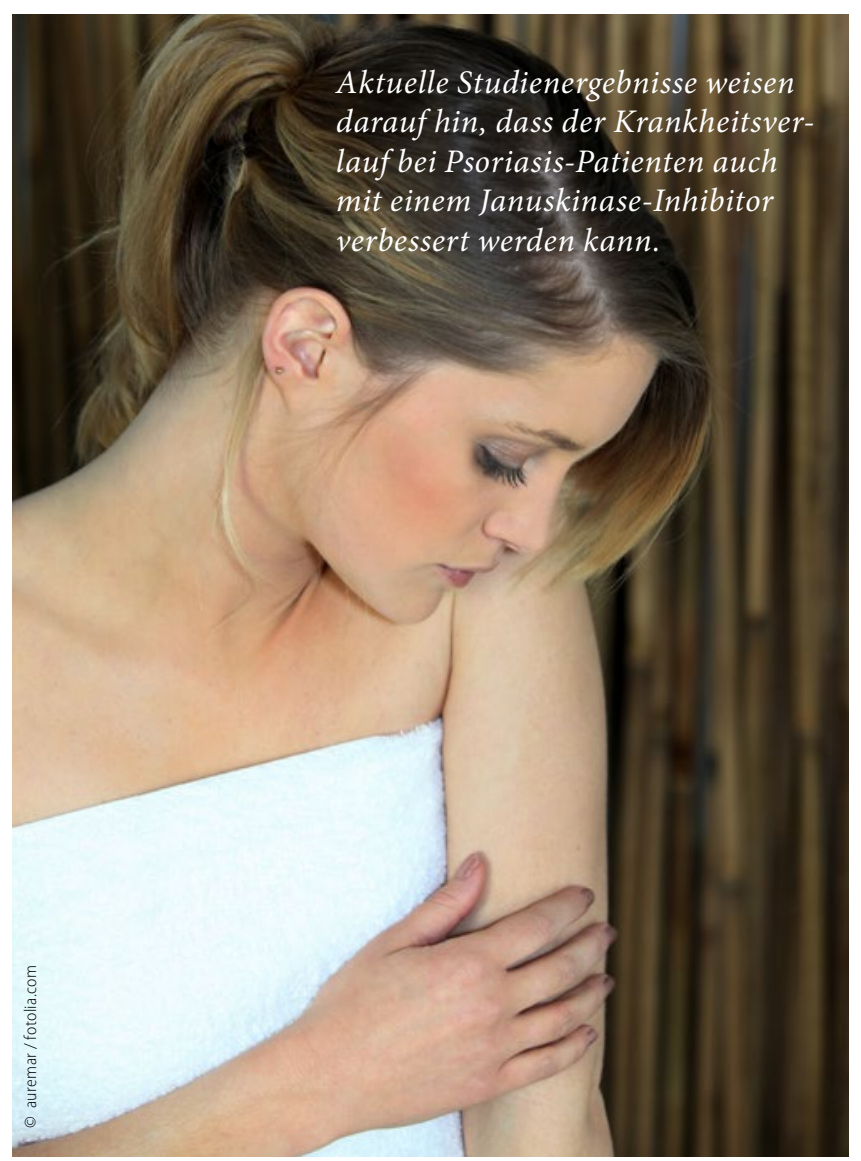

zierte Studie gewinnen. Alle Teilnehmer wiesen eine PlaquePsoriasis auf, die mehr als $10 \%$ der Körperfläche betraf, und waren Kandidaten für eine Phototherapie. Konventionelle systemische Therapieversuche hatten zuvor entweder nicht gewirkt oder es lagen Kontraindikationen bzw. Intoleranzen vor.

330 Patienten nahmen Tofacitinib in einer 5-mg- und 332 in einer 10-mg-Dosis jeweils zweimal täglich ein, 336 Teilnehmer bekamen $50 \mathrm{mg}$ Etanercept subkutan zweimal wöchentlich, 108 Patienten erhielten Placebo. Die meisten Teilnehmer (93\%) beendeten die über zwölf Wochen laufende Studie. Zum Studienende hatte sich bei $5 \%$ der Patienten mit der 5-mg- und bei $6 \%$ mit der 10-mg-Tofacitinib-Dosis der „Psoriasis Area and Severity“(PASI)-Score um mindestens $75 \%$ verbessert, mit Etanercept war das bei $8 \%$ und mit Placebo bei $6 \%$ der Fall. Ein „klares Hautbild“ oder "nahezu klares Hautbild“ im „Physician Global Assessment for Psoriasis“(PGA)-Score erreichten 1\% (Tofacitinib $5 \mathrm{mg}$ ), $2 \%$ (Tofacitinib $10 \mathrm{mg}$ ), $3 \%$ (Etanercept) und $0 \%$ (Placebo) der Patienten. Schwere Nebenwirkungen traten generell selten und in allen Behandlungsgruppen vergleichbar häufig auf; am häufigsten waren Infektionen des Nasopharyngeal- und oberen Respirationstraktes.

\section{Orale Applikation mit Vorteilen}

Diese Studie sei damit die erste Phase-3-Studie, in der eine orale Substanz wie Tofacitinib in der 10-mg-Dosis dieselbe Wirksamkeit und Sicherheit bei Patienten mit mittelschwerer bis schwerer Plaque-Psoriasis gezeigt habe wie ein parenterales Biologikum, schreiben die Studienautoren. Mit der 5-mg-Dosis ließ sich zwar keine statistische Überlegenheit gegenüber Etanercept nachweisen. Doch auch damit habe sich der Krankheitsverlauf bei einem erheblichen Teil der Patienten verbessert, bemerken Bachelez und Kollegen.

\section{Aber: HDL- und LDL-Anstieg beobachtet}

Allerdings sei unter Tofacitinib - wie auch in früheren Studien bei Patienten mit Rheumatoider Arthritis zu sehen war - ein Anstieg des LDL- und HDL-Cholesterins festgestellt worden, führen die Wissenschaftler an. Doch ob und inwieweit Tofacitinib das kardiovaskuläre Risiko beeinflusse, bleibe unklar. Auch deshalb müsse das Sicherheits- und Wirksamkeitsprofil der Substanz in Langzeitstudien bestätigt werden. Wenn dies gelänge, könnte Tofacitinib künftig eine geeignete Behandlungsoption für Patienten mit mittelschwerer bis schwerer Plaque-Psoriasis darstellen, schließen die Studienautoren.

Veronika Schlimpert

Bachelez $\mathrm{H}$ et al. Tofacitinib versus etanercept or placebo in moderate-tosevere chronic plaque psoriasis: a phase 3 randomised non-inferiority trial. The Lancet 2015; online 4. Juni 2015 